\title{
Adaptive Terminal-Modality-Based Joint Call Admission Control for Heterogeneous Cellular Networks
}

\author{
Mahmoud M. Badawy, Salman A. AlQahtani \\ Computer Engineering Department, King Saud University, Riyadh, Saudi Arabia \\ Email: zmahmoudh@yahoo.com,salmanq@ksu.edu.sa
}

Received July 2, 2013; revised August 4, 2013; accepted August 14, 2013

Copyright (C) 2013 Mahmoud M. Badawy, Salman A. AlQahtani. This is an open access article distributed under the Creative Commons Attribution License, which permits unrestricted use, distribution, and reproduction in any medium, provided the original work is properly cited.

\begin{abstract}
The coexistence of different Radio Access Technologies (RATs) requires a need for Common Radio Resource Management (CRRM) to support the provision of Quality of Service (QoS) and the efficient utilization of radio resources. The provision of QoS is an important and challenging issue in the design of integrated services packet networks. Call admission control (CAC) is an integral part of the problem. Clearly, without CAC, providing QoS guarantees will be impossible. There is unfairness in allocation of radio resources among heterogeneous mobile terminals in heterogeneous wireless networks. In this paper, an Adaptive-Terminal Modality-Based Joint Call Admission Control (ATJCAC) algorithm is proposed to enhance connection-level QoS and reduce call blocking/dropping probability. The proposed ATJCAC algorithm makes call admission decisions based on mobile terminal modality (capability), network load, adaptive the bandwidth of ongoing call and radio access technology (RAT) terminal support index. Simulation results show that the proposed ATJCAC scheme reduces call blocking/dropping probability.
\end{abstract}

Keywords: Call Admission Control; Call Blocking; Call Dropping; Next Generation Wireless Network (NGWN); RAT Selection Approaches

\section{Introduction}

Network heterogeneity refers to a combination of multiple wireless networks based on different access technologies (e.g. UMTS, EV-DO, LTE, WiMAX, etc.) coexisting in the same geographical area. Due to the coexistence of different Radio Access Technologies (RATs), Next Generation Wireless Networks (NGWN) are predicted to be heterogeneous in nature. The coexistence of different RATs requires a need for Common Radio Resource Management (CRRM) to support the provision of Quality of Service (QoS) and the efficient utilization of radio resources. With joint radio resource management in NGWN, mobile users will be able to communicate through any of the available radio access technologies (RATs) and roam from one RAT to another, using multimode terminals (MTs) as shown in Figure 1 [1-3].

Next generation wireless cellular networks, including $3 \mathrm{G}$ and $4 \mathrm{G}$ technologies are envisaged to support more mobile users and variety of high-speed Wireless Multimedia Services (WMSs). A WMS enables the simultaneous transmission of voice, data, text and images through radio links by means of the new wireless tech- nologies. Different WMSs have diverse bandwidth and Quality of Service (QoS) requirements from their users that need to be guaranteed by wireless cellular networks. In wireless cellular networks, user's QoS requirements can be quantitatively expressed in terms of probabilistic connection-level QoS parameters such as new call blocking probability (NCBP) and handoff call dropping probability (HCDP) [4]. The NCBP is the probability of a new arriving call being rejected while the HCDP is the probability that an accepted call is terminated before the completion of its service, i.e., the probability that a handoff attempt fails [4].

Provisioning connection-level QoS in wireless cellular networks becomes complex due to 1) The limited radio link bandwidth, and 2) The high rate of handoff events as the next generation of wireless cellular networks will use micro/pico cellular architectures in order to provide higher capacity. Therefore, one of the most important connection-level QoS issues is how to reduce/control handoff drops due to lack of available resources in the new cell, since mobile users should be able to continue their ongoing connections. Since it is practically impossible to completely eliminate handoff drops, the best one 


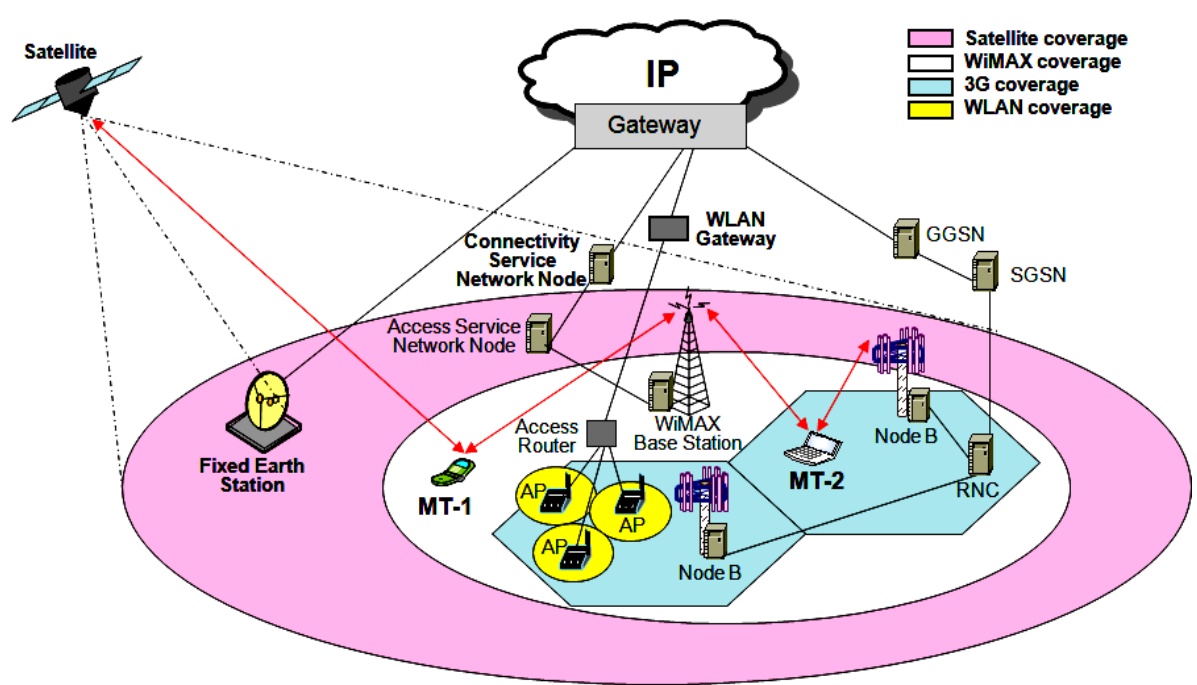

Figure 1. The coexistence of different RATs.

can do is to provide some forms of probabilistic QoS guarantees by keeping HCDP below a predetermined value [5].

In the $3 \mathrm{G}$ and beyond wireless systems, multimedia services such as voice, video, data, and audio are to be offered with various quality-of-service (QoS) profiles. Hence, more sophisticated call admission control (CAC) schemes are developed to cope with these changes. Traffic of admitted calls is then controlled by other RRM techniques such as scheduling, handoff, power, and rate control schemes.

RAT selection algorithms are part of the CRRM algorithms. Simply, their role is to verify if an incoming call will be suitable to fit into a heterogeneous wireless network, and to decide which of the available RATs is most suitable to fit the need of the incoming call and admit it. Guaranteeing the requirements of QoS for all accepted calls and at the same time being able to provide the most efficient utilization of the available radio resources is the goal of RAT selection algorithm. Call admission control is a key element in the provision of guaranteed quality of service in wireless networks. The design of call admission control algorithms for mobile cellular networks is especially challenging given the limited and highly variable resources, and the mobility of users encountered in such networks.

Generally, CAC algorithms are triggered by any of the following events: New call arrival and handoff call arrival. The normal call admission control algorithms do not provide a solution to fit a heterogeneous wireless network. Therefore, there is a need to develop RAT selection algorithm in addition to Call admission control. This guarantees a term called Joint call admission control (JCAC) algorithm.

In this paper, an Adaptive-Terminal Modality-Based Joint Call Admission Control (ATJCAC) algorithm is proposed to enhance connection-level QoS and reduce call blocking/dropping probability. The ATJCAC scheme is designed to simultaneously achieve the following objectives in heterogeneous cellular networks:

1) Ensure fairness in allocation of radio resources among heterogeneous mobile terminals;

2) Adapt the bandwidth of ongoing calls to improve connection-level QoS;

3) Guarantee the QoS requirement of all admitted calls;

4) Prioritize handoff calls over new calls.

The rest of this paper is organized as follows. The related work is presented in the next section. In Section 3, the system model is described. The proposed adaptiveTJCAC scheme is presented in Section 4. In Section 5, result discussions of the proposed scheme are provided. Finally, the conclusion of this research is presented in Section 6.

\section{Literature Review}

A number of RAT selection algorithms including initial RAT selection and vertical handover have been proposed in the literature for heterogeneous wireless networks [1,2, 6-14]. Reference [14] presents a good revision on these algorithms. Each one has its benefits and limitations. O. E. Falowo et al. in paper [1] review the recent call admission control algorithms for heterogeneous wireless networks. The benefits and requirements of JCAC algorithms are discussed. The authors examine eight different approaches for selecting the most appropriate RAT for incoming calls in HWN and classify the JCAC algorithms based on these approaches. The advantages and disadvantages of each approach are discussed. The same authors in [2] propose a JCAC algorithm which considers the users preference in making an admission decision and 
a specific case where the user prefers to be served by the RAT which has the least service cost is modeled and evaluated. In [6] a JCAC scheme for multimedia traffic that maximizes the overall network revenue with QoS constraints over coupled WLAN and CDMA cellular network is considered. X. G. Wang et al. in [7] proposed an adaptive call admission control for integrated cellular and WLAN network. In this proposed scheme, call admission decisions are based on requested QoS and availability of radio resources in the considered RATs. D. Karabudak et al. in [8] proposed a call admission control scheme for the heterogeneous network using genetic algorithm. The objectives of the scheme are to achieve maximum wireless network utilization and meet QoS requirements. A network capacity policy based joint admission controller is presented by $\mathrm{K}$. Murray et al. in $[9,10]$. D. Qiang et al. in [11] proposed a joint admission control scheme for multimedia traffic that exploits vertical handoffs as an effective tool to enhance radio resource management while guaranteeing handoff users QoS requirements. The network resources utilized by the vertical handoff user are captured by a link utility function. X. Li et al. in [12] proposed an efficient joint session admission control scheme that maximizes overall network revenue with QoS constraints over both the WLAN and the TD-SCDMA cellular networks. In [13], the authors proposed a call admission control reservation algorithm that takes resource fluctuations into consideration. They considered two types of applications denoted by wide-band and narrow band. The performance of the algorithm was modeled through a queuing theory approach and its main performance measures are compared with a conventional algorithm through simulation. The authors in [14] proposed an algorithm, which incorporates traditional Admission Control (AC) and Wiener Process (WP)-based prediction algorithms to determine when to carry out access service network gateway relocation.

Gelabert et al. in [15] presented a Markovian approach to RAT selection in heterogeneous wireless networks. They developed an analytical model for RAT selection algorithms in a heterogeneous wireless network comprising GSM/EGDE and UMTS. The proposed algorithm selects just one RAT for each call. In [16], a service-class based JCAC algorithm was proposed. it admits calls into a particular RAT based on the class of service, such as voice, video streaming, real-time video, web browsing, etc. in [17], a terminal-modality-based JCAC scheme was proposed. It consists of two main components: joint call admission controller and band-width reservation unit.

\section{System Model and Assumptions}

We consider a heterogeneous cellular network which consists of $\mathrm{J}$ number of RATs with co-located cells. A typical example of a heterogeneous wireless network, adapted from [16] is shown in Figure 2. In the heterogeneous network, radio resources are jointly managed. Cellular networks such as GSM, UMTS (3G) and LTE can have the same and fully overlapped coverage, which is technically feasible, and may also save on installation costs $[18,19]$. Let $H$ denote the set of all available RATs in the heterogeneous wireless network.

Then, $H$ is given as follows:

$$
H=\{\mathrm{RAT} 1, \mathrm{RAT} 2, \cdots \mathrm{RAT} j\}
$$

where $J$ is the total number of RATs in the heterogeneous cellular network. The heterogeneous cellular network supports $k$-classes of calls, and each RAT in set $H$ is optimized to support certain classes of calls. Let $H_{i}\left(H_{i} \subseteq H\right)$ denote the set of RATs which can support class- $i$ calls in the heterogeneous cellular network, and let $h_{i}\left(h_{i} \subseteq h\right)$ denote the set of indices of all RAT $j$ which belong to $H_{i}$, where $h=\{1,2, \cdots, J\}$. Furthermore, let $J_{i}\left(J_{i} \leq J\right)$ denote the total number of RATs that can support class- $i$ calls. Let $D_{j}\left(D_{j} \subseteq D\right)$ denotes the set of call classes that can be supported by $\operatorname{RAT} j(j=1,2, \cdots, J)$ where $D=\{$ class- 1 , class- $2, \cdots$, class- $k\}$. Note that the idea that different networks support different classes of calls is true in reality. For example, LTE and UMTS network can support video streaming whereas GSM network cannot support video streaming.

Each cell in $\operatorname{RAT} j(j=1, \cdots, J)$ has a total of $B j$ basic bandwidth units $(b b u)$. The physical meaning of a unit of radio resources (such as time slots, code sequence, etc.) is dependent on the specific technological implementation of the radio interface [20]. However, no matter which multiple access technology (FDMA, TDMA, WCDMA or OFDMA) is used, system capacity could be interpreted in terms of effective or equivalent bandwidth [21-22]. Therefore, this research refers to the bandwidth of a call as the number of $b b u$ that is adequate for guaranteeing the desired QoS for the call, which is similar to the approach used for homogeneous networks in [22,23].

It is assumed that packet-level QoS is stochastically assured by allocating at least the minimum effective bandwidth required to guarantee a given maximum probability on packet drop, delay, and jitter. The approach used is to decompose a heterogeneous cellular network into groups of co-located cells as shown in Figure 3.

For example, cell $1 a$ and cell $2 a$ form a group of colocated cells. Similarly, cell $1 b$ and cell $2 b$ form another group of co-located cells, and so on. When a mobile user with an ongoing call is moving outside the coverage area of a group of co-located cells, the call must be handed over to one of the cells that can support the call in the neighboring group of co-located cells. For example, in the two-class three-RAT heterogeneous cellular network 


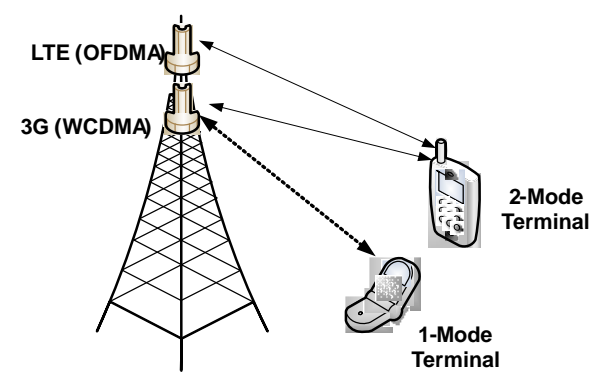

Figure 2. A typical two-RAT heterogeneous cellular network with co-located.

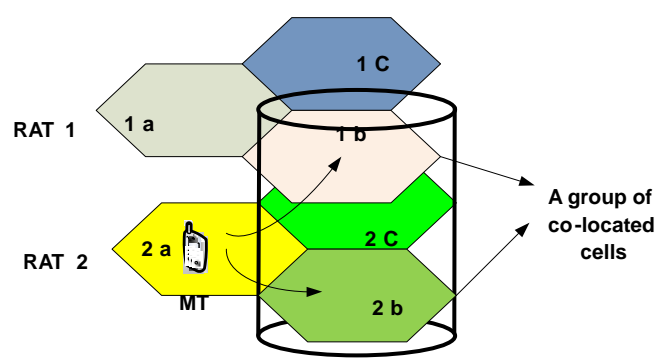

Figure 3. Two-RAT heterogeneous cellular networks with co-located cells.

illustrated in Figure 3, an ongoing class- 1 call can be handed over from cell $2 a$ to cell $2 b$, or from cell $2 a$ to cell $1 b$. Note that hand-off comprises both horizontal and vertical handoffs.

The correlation between the groups of co-located cells results from handoff connections between the cells of corresponding groups. Under this formulation, each group of co-located cells can be modeled and analyzed individually. Therefore, a single group of co-located cells is considered in this research. The heterogeneous network supports $K$ classes of calls. Each class is characterized by bandwidth requirement, arrival distribution, and channel holding time. Each class- $i$ call requires a discrete bandwidth value, $b_{i, w}$ where $b_{i, w}$ belongs to the set $B_{i}=$ $\left\{b_{i, w}\right\}$ for $i=1,2, \cdots, K$ and $w=1,2, \cdots, W_{i} . W_{i}$ is the number of different bandwidth values that a class- $i$ call can be allocated. $b_{i, 1}$ (also denoted as $b_{i, \text { min }}$ ) and $b_{i, W i}$ (also denoted as $\left.b_{i, \max }\right)$ are, respectively, the minimum and maximum bandwidth that can be allocated to a class- $i$ call. Note that $b_{i, w}<b_{i,(w+1)}$ for $i=1,2 \cdots K$ and $w=1$, $2 \cdots\left(W_{i}-1\right)$.

The requested bandwidth of class- $i$ call is denoted by $b_{i, \text { req }}$, where $b_{i, \text { req }} \in B_{i}$. Let $m_{i, j}$ and $n_{i, j}$ denote, respectively, the number of new call of class- $i$ and handoff call of class- $i$, in RAT- $j$. with $1 \leq c \leq m_{i, j}$ (for new calls) and

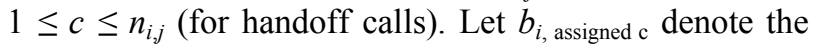
bandwidth assigned to call $c$ of class $-i$ in RAT- $j$ in the group of co-located cells, where $b_{i \text {, assigned c }} \in B_{i}$. A call $c$ of class $-i$ is degraded if $b_{i \text {, assigned c }}<\mathrm{b}_{\mathrm{i} \text {,req }}$ whereas the call is upgraded if $b_{i}$, assigned c $>b_{i}$, req. If a class of calls (i.e., class- $i$ calls) requires a fixed number of $b b u$ (i.e. constant bit-rate service), it becomes a special case in our model in which $b_{i, \text { min }}=b_{i, \text { max }}$ and the set $B_{i}$ has only one element. However, it will not be possible to upgrade or degrade this class of calls.

We define the following terms commonly used in the literature to be used throughout this paper.

1) Call holding time: It is duration of the requested call connection. This is a random variable which depends on the user behavior (call characteristics).

2) Cell residency time: It is amount of time during which a mobile terminal stays in a cell during a single visit. Cell residency is a random variable which depends on the user behavior and system parameters, e.g. cell geometry.

3) Channel holding time: How long a call which is accepted in a cell and is assigned a channel will use this channel before completion or handoff to another cell? This is a random variable which can be computed from the call holding time and cell residency time and generally is different for new calls and handoff calls.

Following are the general assumptions in the studied cellular networks. The New call arrival of class-i arrive is assumed to follow Poisson process with rate $\lambda_{i}^{n}, n$ denoted to new call. Handoff call of class-i arrive according to Poisson process with rate $\lambda_{i}^{h}, \mathrm{~h}$ denoted to handoff call. Call holding time (CHT) of class-i is assumed to exponential distribution with mean $\mu_{c i}^{-1}$. Cell residence time (CRT) is assumed to follow an exponential distribution with mean $\mu_{h i}^{-1}, h$ denoted to handoff rate. Channel holding time for call of class-i is assumed to exponential distribution with mean $\mu_{i}^{-1}$ where $\mu_{i}=\mu_{c i}+\mu_{h i}$.

\section{Proposed Adaptive TJCAC Scheme}

This section describes the proposed adaptive terminalmodality-based JCAC scheme. In fact, the joint call admission control (JCAC) algorithm is one of the RRM algorithms. The basic function of JCAC algorithms is to decide whether an incoming call can be accepted or not. They also decide which of the available radio access technology is most suitable to accommodate the incoming call. Figure 4 shows call admission control procedure in heterogeneous cellular networks.

When these mobile terminals make a call, then they will send a service request to the JCAC algorithm. The JCAC scheme, which executes the JCAC algorithm, will then select the most suitable RAT for the incoming call.

Figure 5 illustrates the problem of unfairness in radio resource allocation in a three-RAT heterogeneous wireless network when terminal modality is not considered in making RAT selection decisions. Assume that 1) all the three RATs have equal capacity; 2) all the arriving calls belong to the same class; and 3) each RAT can support only two calls. Figure 5 shows six consecutively arriving calls (1 to 6$)$ in the heterogeneous wireless network. A load-balancing JCAC scheme, for instance, will admit the 


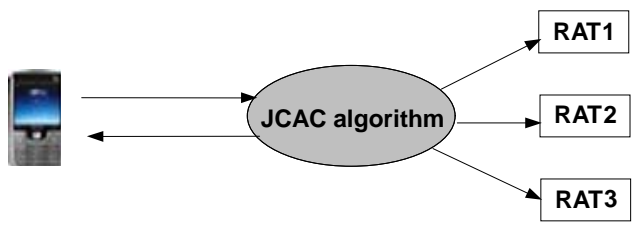

Figure 4. JCAC procedure in heterogeneous cellular networks.

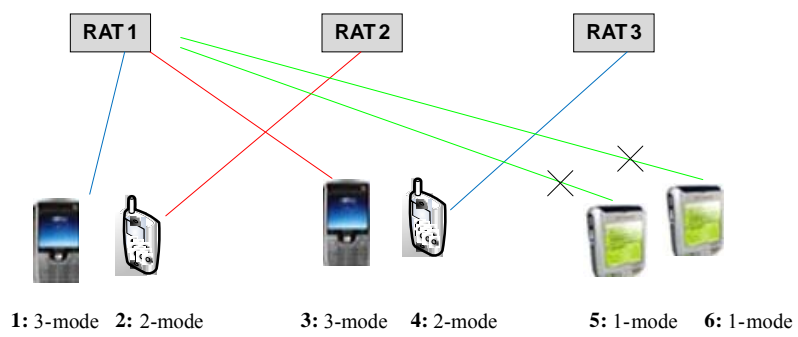

Figure 5. Unfair allocation of radio resources among heterogeneous mobile terminals.

first incoming call (call 1 from a triple-mode terminal) into RAT 1. It will admit call 2 (from a dual-mode terminal) into RAT 2, admit call 3 (from a triple-mode terminal) into RAT 3, and admit call 4 (from a dual-mode terminal into RAT1).

The JCAC scheme cannot admit the fifth incoming call (call 5) into RAT 2 or RAT 3 be-cause call 5 is from a single-mode terminal that is sup-ported only by RAT 1 . The JCAC scheme will then try to admit call 5 into RAT 1. Call 5 will be blocked in RAT 1 because it is already fully loaded (maximum of two calls). In a similar manner, the sixth incoming call (call 6) from a single-mode terminal that is supported by RAT 1 only, will be blocked.

In order to reduce this problem of unfairness in allocation of radio resources and also enhance QoS and system utilization among heterogeneous terminals in heterogeneous wireless networks, adaptive-terminal modalitybased JCAC (ATJCAC) scheme is proposed for heterogeneous wireless networks. ATJCAC consists of the following three components: joint call admission controller, band-width reservation unit and bandwidth adaptation controller. The main component of the proposed scheme is shown in Figure 6.

The following are the descriptions of these main components.

\subsection{The Joint Call Admission Controller}

The joint call admission controller implements the JCAC algorithm. The basic function of the JCAC algorithm is to make call admission decisions and guarantee fairness among the different heterogeneous terminals in the heterogeneous wireless network. The proposed JCAC algorithm makes RAT selection decisions based on the modality of the mobile terminal initiating a call, the terminal support index of each RAT that can support the call, and the current load in the available RATs.

Terminal support index of a RAT indicates the ratio of terminals supported by the RAT to the total terminals registered in the heterogeneous wireless network. Terminal support index of $\operatorname{RAT} j\left(R^{J}\right)$ is defined as follows:

$$
R^{j}=T^{J} / T, 0<R^{j} \leq 1
$$

where $T^{j}$ is the number of mobile terminals that have an interface for RAT-j (i.e. that can be admitted into RAT-j), and $T$ is the total number of mobile terminals registered in the network.

During a call setup, a mobile terminal initiating a call sends a service request to the joint call admission control which implements the JCAC algorithm. The JCAC algorithm is part of the joint resource management entity of the heterogeneous wireless network. The location of the joint resource management entity depends on the specific network architecture deployed by the network operator. The service request contains the call type, capability of terminal (number and types of network supported by the terminal), service class, and bandwidth requirements. Figure 7 is the flow chart of the proposed JCAC algorithm. As shown in Figure 7, $x_{i j}$ and $y_{i j}$ represent the residual $b b u$ available for new and handoff class- $i$ calls, respectively, in RAT-j. $L_{j}$, and $T^{j}$ represent the current load and Terminal support index of RAT-j, respectively. $H_{i}$ is the set of RATs that can support an incoming class- $i$ call, and the mobile terminal (based on terminal modality) initiating the call. $h_{i}$ is the set of indexes of RATs that belong to set $H_{i}$.

As shown in Figure 7, whenever a class- $i$ call arrives from a y-mode terminal in the heterogeneous wireless network, the JCAC algorithm determines the set $H_{i}$ of RAT- $j$ that can support the class- $i$ call and that can support the mobile terminal initiating the call (based on terminal modality). The JCAC algorithm then sorts the RATs in set $H_{i}$ in increasing order of their terminalsupport index $\left(R_{j}\right)$. Starting from the RAT with the lowest terminal-support index, the JCAC algorithm attempts to allocate the maximum $b b u$ for this call (i.e., set $b_{i, \min }=$ $b_{i, \text { max }}$ ) provided that the available $b b u$ in the selected RAT is greater than or equal to $b_{i \text {,max }}$. If the available $b b u$ in the selected RAT is less than $b_{i \text {,max }}$ but greater than or equal to $b_{i, \text { req }}$, the call will be assigned a bandwidth between $b_{i \text {,req }}$ and $b_{i, \text { max }}$. If the available $b b u$ is less than $b_{i, \text { req }}$ but greater than or equal to $b_{i, 1}\left(b_{i, \min }\right)$, the call will be assigned a bandwidth between $b_{i, 1}$ and $b_{i, \text { req. }}$

If the available $b b u$ in RATs with the lowest terminal-support index is less than $b_{i, 1}$, BA algorithm (BAA) will be invoked to reduce the bandwidth of some ongoing call(s) in the chosen RAT. If the available $b b u$ is still less than $b_{i, 1}$, the next available RAT with the second-lowest terminal support index will be selected for the call, and so on. If two or more RATs that can support the incom- 


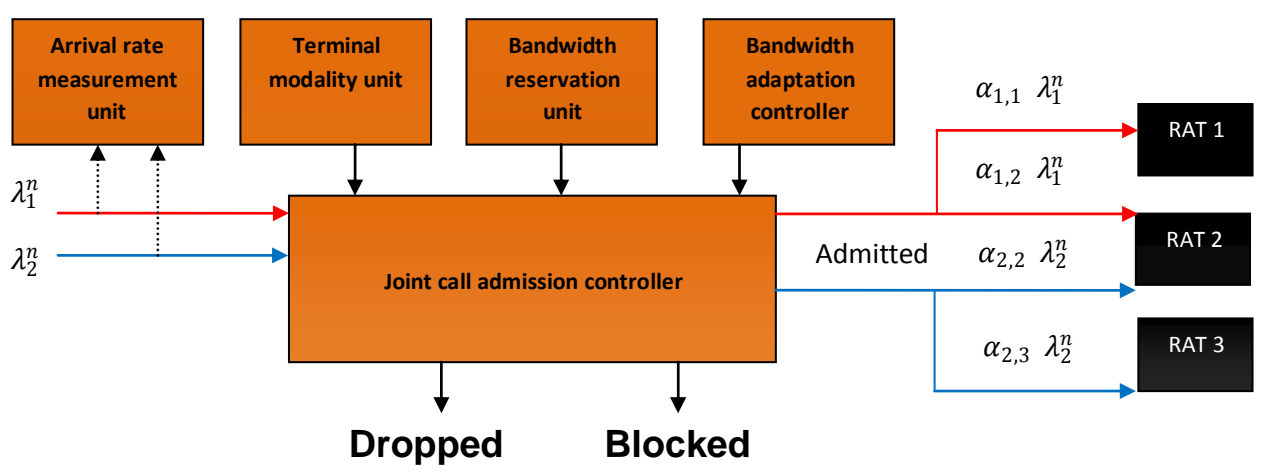

Figure 6. Component of the proposed ATJCAC scheme.

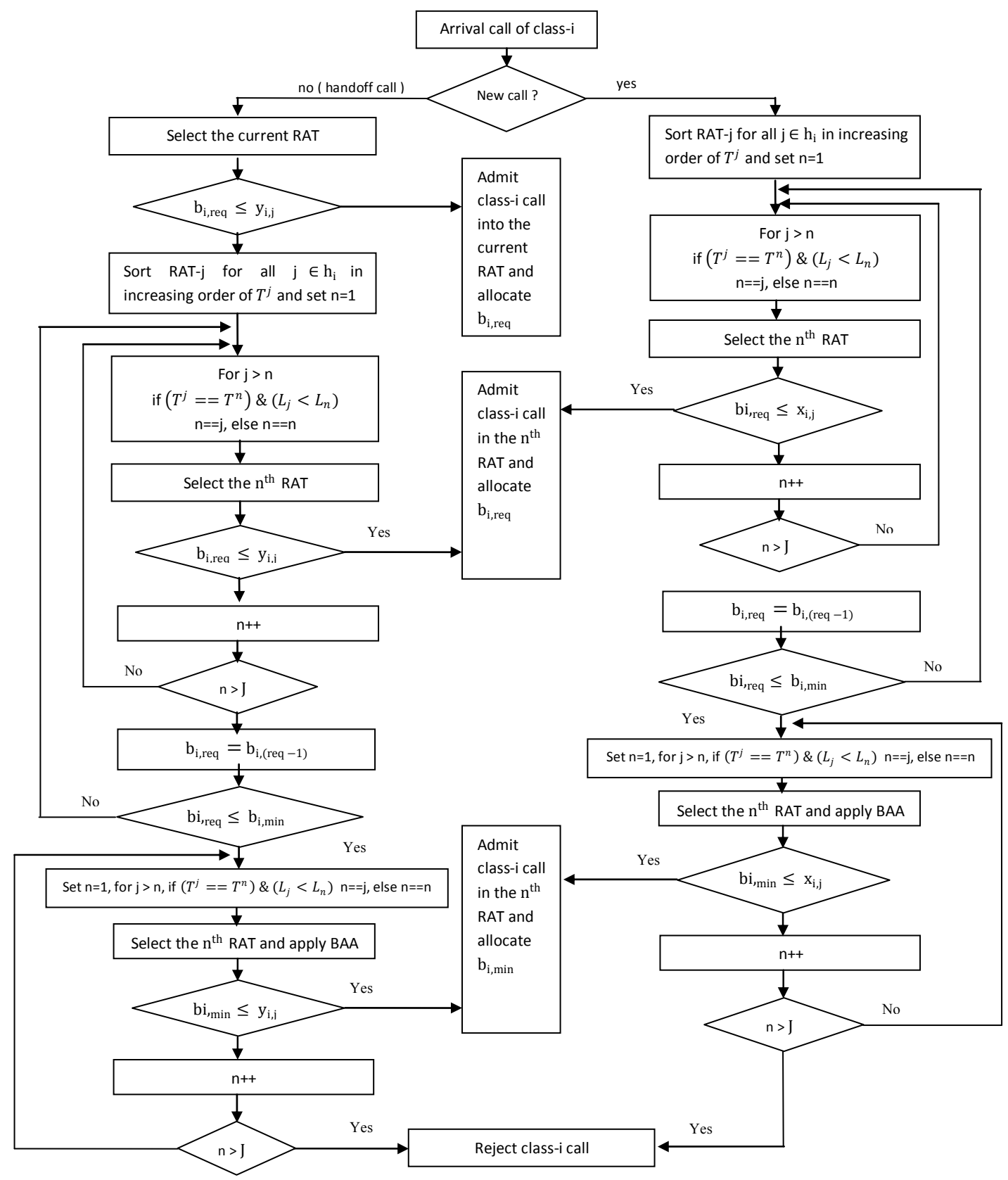

Figure 7. Flow chart of the proposed ATJCAC algorithm 
ing call have the same low terminal-support index, the least loaded of the two or more RATs will be selected for the incoming call.

The class- $i$ call is rejected if none of the RATs in set $H_{i}$ has enough basic bandwidth units $(b b u)$ to accommodate the call. By attempting to admit an incoming call into the RAT with the lowest terminal-support index that can support the class of the call, the proposed ATJCAC scheme reserves other RATs with a higher terminalsupport index for calls from low-capability terminals, thereby reducing the blocking probability of such calls.

Figure 8 illustrates how the proposed JCAC scheme reduces the problem of unfairness in allocation of radio resources among heterogeneous mobile terminals. As show in Figure 8, and using Equation (1), the terminal support index of RAT 1 , RAT 2 , and RAT 3 are $6 / 6,4 / 6$, and $2 / 6$ respectively. Note that terminal support index is calculated based on the number of terminals registered in the heterogeneous wireless network, and it is assumed that there only six terminals in this example.

As shown in Figure 8, the first incoming call (call 1 from a triple-mode terminal) can be admitted into any of the three RATs. The proposed ATJCAC will select the RAT with the lowest terminal support index (RAT 3), thereby reserving RAT 1 and RAT 2 for calls from lowcapability mobile terminals. The second incoming call (call 2 from a dual-mode terminal) can be admitted into RAT 1 or RAT 2, The ATJCAC scheme selects RAT 2, which has the lower terminal support index. The third incoming call (call 3 is from a triple mode terminal) can be admitted into any of the three RATs. The ATJCAC selects RAT 3 with the lowest terminal support index. The fourth call is admitted into RAT 2. The fifth and sixth calls are then admitted into RAT 1, which has the highest terminal support index. Thus the problem of unfairness in allocation of radio resources is reduced.

\subsection{Bandwidth Reservation Unit}

In order to maintain lower handoff calls over new calls, we use different a threshold-based bandwidth reservation unit. The policy reserves bandwidth for aggregate handoff calls, thus gives them priority over new calls. The policy also prioritizes among different classes of handoff calls according to their QoS constraints by assigning a series of bandwidth thresholds $t_{0, j}, t_{1, j}, \cdots, \cdots, t_{k, j}$ for handoff calls such that

$$
t_{0, j} \leq t_{1, j} \leq \cdots \leq t_{i, j} \leq t_{(i+1), j} \leq t_{k, j}=B_{j, \forall j}
$$

where $t_{0, j}$ : the total $b b u$ available in $\operatorname{RAT} j$ for new call (is the threshold after which new calls will be rejected in RAT $-j$ ) and $t_{i, j}$ denotes the total number of $b b u$ available for handoff class- $i$ calls in RAT $j . B_{j}$ denotes the total number of $b b u$ available in RATj. When given any val-

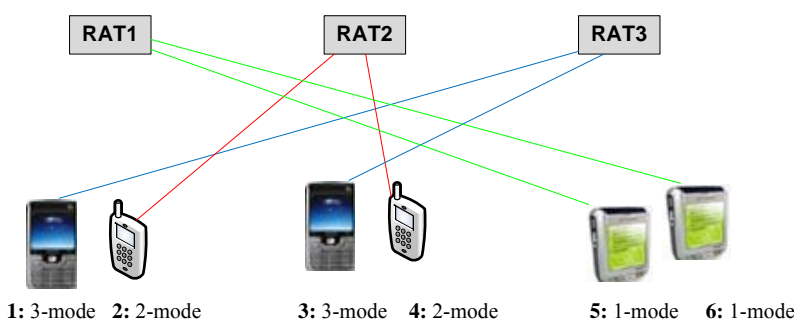

Figure 8. Fair allocation of radio resources among heterogeneous mobile terminals.

ues of threshold (ex. $t_{0, j}$ ), there exist optimal values of $\alpha_{i, j}$ and $\beta_{i j}\left(j=1, \cdots, J, i \in d_{i, j}\right.$ where $d_{i, j}$ denotes the set of indices of all class- $i$ calls that can be supported by RAT-j) that minimizes the overall blocking probability in the heterogeneous wireless network. Figure 9 shows the band-width reservation unit for a two-class two-RAT heterogeneous cellular network $j$ noting that $C_{j}=C_{h i j}$ and $t_{0, j}=C_{n i j}$

\subsection{Bandwidth Adaptation/Reallocation Controller}

The bandwidth adaptation controller executes the BAA which is triggered when a new call arrives or when a call is completed. Most multimedia applications are adaptive. For example, voice can be encoded at $16 \mathrm{kbps}, 32 \mathrm{kbps}, 64$ kbps, and $128 \mathrm{kbps}$ by choosing appropriate encoding mechanisms. Similarly, video applications can be made rate adaptive by using, for instance, a layered coding method. In layer coding method, the lowest layer (i.e., the base layer) contains the critical information for decoding the image sequence at its minimum visual quality. Additional layers provide increasing quality. All these encoded layers may be transmitted when the network is underutilized. However, when the network resources are being fully utilized, only based layer(s) which contain critical information may be transmitted.

As an illustration, if one would watch a 30-minute video clip encoded at $256 \mathrm{kbps}$ and $64 \mathrm{kbps}$ respectively. At $256 \mathrm{kbps}$, one will see better pictures with better resolution than at $64 \mathrm{kbps}$. Therefore, the bandwidth adaptation affects the quality of the real-time applications rather than the transmission time. However, the minimum requested QoS is maintained by ensuring that the $b b u$ of the calls are not degraded below the required minimum.

Under conditions of heavy traffic load, i.e. the sum of the requested bandwidth exceeds the unused bandwidth capacity so that not all the requests can be completely served, the role of bandwidth adaptation technique are essential. These algorithms are needed to reduce the requested or already connected call bandwidth allocation. In designing the algorithm, we assume that a service with degraded QoS is better than an outright rejection of service requests. The quality grade is determined by the 
amount of Acceptable Bandwidth level (ABL).

The Bandwidth Reallocation (i.e. degradation or upgrading of resource allocation) module is deployed to reallocate the bandwidth capacity. The reallocation forms the crust of the scheme/algorithm. The strategy implemented is by cohesively coupling lower and priority calls in a trade-off manner. In the event of a new call or handoff from a higher priority traffic encountering insufficient bandwidth level, sustaining the call is compulsory in the presence of hybrid traffic. Bandwidth of lower priority connections (i.e. non-real-time and real-time VBR traffic) are decreased to the level of streams of the lowest priority or the highest ABL. In the event of a vise versa conditions, bandwidth of lower priority traffic are able to be increased to a maximum level of the highest priority level. The process of reallocating may be involve either an upgrade or degrade of the bandwidth allocation based on the ABL.

The ABL is obtained by subtracting the maximum required bandwidth with the minimum required bandwidth. The difference is called the degradable range/spectrum. The degradable spectrum is further divided into $N$ (i.e. where $N=1,2 \cdots n$ ) levels, called micro-ABLs as shown in Figure 10.

The concept of bandwidth allocation as a discrete component is applied into the structuring and derivation of the micro-ABLs. Subsequent to this theory, the bandwidth allocation for the micro-ABLs form the discrete set $\mathrm{B}=\left\{\mathrm{BW}_{\min }, \mathrm{BW}_{(\min +1)}, \mathrm{BW}_{(\min +2)}, \cdots, \mathrm{BW}_{\mathrm{avg}}, \cdots, \mathrm{BW}_{\max }\right\}$ where $\mathrm{BW}_{\min }$ is the minimum bound, $\mathrm{BW}_{\text {avg }}$ is the average bound and $\mathrm{BW}_{\max }$ is the maximum bound for bandwidth allocation.

The computation and deployment of bandwidth reallocation consumes an amount of time capable of jeopardizing the probability of a mobile to continue is con-

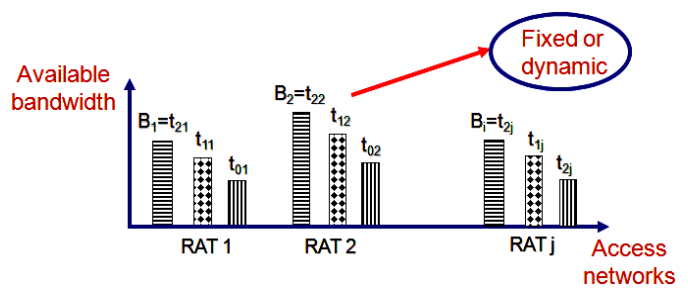

Figure 9. Fair allocation of radio resources among heterogeneous mobile terminals.

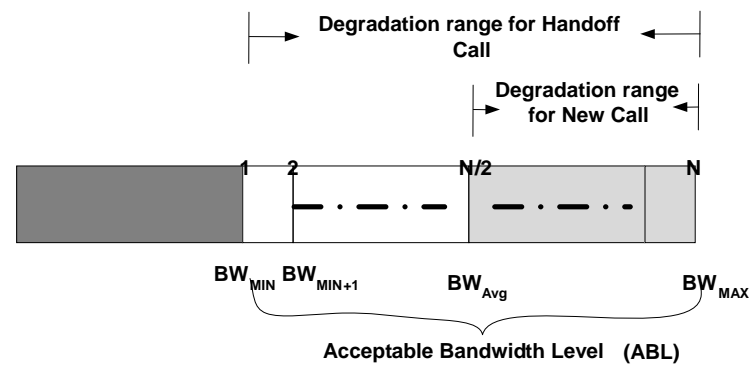

Figure 10. ABL and Micro-ABLs range/spectrum. nection. Thus, to avoid this time delay, the process of bandwidth reallocation is carried out in a distributed manner. Each Base Station (BS) does the computation process independent from other BSs. An important prerequisite is to ensure that the computation process of bandwidth reallocation algorithm should be completed before the system does the real bandwidth reallocation of the ongoing connections in the network.

\section{Results and Discussion}

In this section, the performance of the proposed Adaptive-terminal-modality based JCAC scheme is evaluated with respect to New Call Blocking Probability (NCBP) and Handoff Call Dropping Probability (HCDP), using a one-class three-RAT heterogeneous wireless network supporting heterogeneous mobile terminals. A new call from i-mode terminal is blocked in the group of co-located cells if none of the available RATs supported by i-mode terminal has enough $b b u$ to accommodate the new call. NCBP is the average number of blocked new call over all arrived new call. A handoff call from i-mode terminal is dropped in the group of co-located cells if none of the available RATs supported by i-mode terminal has enough $b b u$ to accommodate the handoff call. HCDP is the average number of dropped new call over all arrived handoff call is known as HCDP.

A numerical simulation is conducted using $\mathrm{C}++$ program and MatLab. The following system parameters are used: $C_{1}=C_{2}=C_{3}=10, t_{01}=t_{02}=t_{03}=5, b_{1}=\{1,2$, $3\}, \mu_{1}=0.5, \lambda_{1}^{n}=[1,5], \lambda_{1}^{h}=0.5 \lambda_{1}^{n}$. In order to investigate the performance of the proposed ATJCAC scheme, two scenarios with different combinations of heterogeneous terminals are examined.

- First scenario (Terminals Dominated by 1-Mode Terminals) $1 \mathrm{M}: 2 \mathrm{M}: 3 \mathrm{M}=50: 25: 25:$ is 1 -mode $=50$ terminal, 2 -mode $=25$ terminal, and 3 -mode $=25$ terminals.

- Second scenario (Terminals Dominated by 2-Mode Terminals) $1 \mathrm{M}: 2 \mathrm{M}: 3 \mathrm{M}=25: 50: 25: 1$-mode $=25$ terminal, 2 -mode $=50$ terminal, and 3-mode $=25$ terminals.

For each of the two scenarios, the performance of the proposed ATJCAC scheme is compared with the performance of two other JCAC schemes namely, the Terminal modality-based JCAC scheme ("NATJCAC" scheme) and the service-class-based JCAC scheme ("SJCAC" scheme). Results obtained from the two scenarios are discussed in the following subsections. In all figures we use letter "A" to indicate ATJCAC, "N" to indicated NATJCAC and letter "S" to indicate SJCAC.

\subsection{First Scenario: $1 M: 2 M: 3 M=50: 25: 25$}

Figure 11 shows the effect of varying the call arrival rate 
on the NCBP $(\mathrm{Pb})$ of 1-mode, 2-mode, and 3-mode terminals for SJCAC, NATJCAC and the proposed ATJCAC. As shown in Figure 11, $\mathrm{Pb}$ for the three JCAC schemes and three classes of terminals increases with an increase in arrival rate for three JCAC schemes. This is expected. However, for 1-mode terminals, $\mathrm{Pb}$ of the NATJCAC scheme is lower than the corresponding $\mathrm{Pb} 1$ of the SJCAC schemes. The NATJCAC scheme is able to reduce the $\mathrm{Pb}$ by admitting most of the calls from 3-mode terminals into RAT 3, which has the lowest terminal support index, thereby reserving RAT 1 and RAT 2 for calls from 1-mode and 2-mode terminals, respectively. However, the reduction in 1 -mode terminals $\mathrm{Pb}$ for NATJCAC scheme is at the expense of the $\mathrm{Pb} 1$ of calls from 3-mode terminals. It can be seen that for 3-mode terminals, the $\mathrm{Pb}$ of the NATJCAC scheme is higher than the corresponding $\mathrm{Pb}$ of the SJCAC schemes.

The NCBP for the ATJCAC scheme is always less than the corresponding NCBP for the NATJCAC scheme. Note that lower NCBP of the ATJCAC scheme implies that its connection-level QoS is better than that of the NATJCAC scheme. The reason why the NCBP of the ATJCAC scheme is less than the NATJCAC scheme is as follows. When the total $b b u$ allocated to new calls is being fully utilized, incoming new calls are rejected by the NATJCAC scheme whereas the ATJCAC scheme adapts (degrades) the bandwidth of some of the ongoing adaptive calls to free just enough $b b u$ to accommodate the incoming new calls. Consequently, the NCBP of the ATJCAC is less than that of the NATJCAC. However, an adaptive class- $i$ call is never degraded below the minimum $b b u$ necessary to guarantee its minimum QoS requirements. For SJCAC the $\mathrm{Pb}$ for one-mode terminals is very high whereas $\mathrm{Pb}$ for two-mode and three-mode terminals is very low. Thus, one-mode terminals are treated unfairly by the SJCAC scheme.

Figure 12 shows the effect of varying the call arrival rate on the HCDP (Pd) of 1-mode, 2-mode, and 3-mode terminals for SJCAC, NATJCAC and the proposed ATJCAC.

As shown in Figure 12, Pd for the three JCAC schemes and three classes of terminals increases with an increase in arrival rate for three JCAC schemes. This is expected. However, for 1-mode terminals, Pd of the NATJCAC scheme is lower than the corresponding Pd of the SJCAC schemes. The NATJCAC scheme is able to reduce the Pd by admitting most of the calls from 3-mode terminals into RAT 3, which has the lowest terminal support index, thereby reserving RAT 1 and RAT 2 for calls from 1-mode and 2-mode terminals, respectively. However, the reduction in 1-mode terminals Pd for NATJCAC scheme is at the expense of the $\mathrm{Pd}$ of calls from 3-mode terminals. It can be seen that for 3-mode terminals, the $\mathrm{Pd}$ of the NATJCAC scheme is higher than the corresponding Pd of

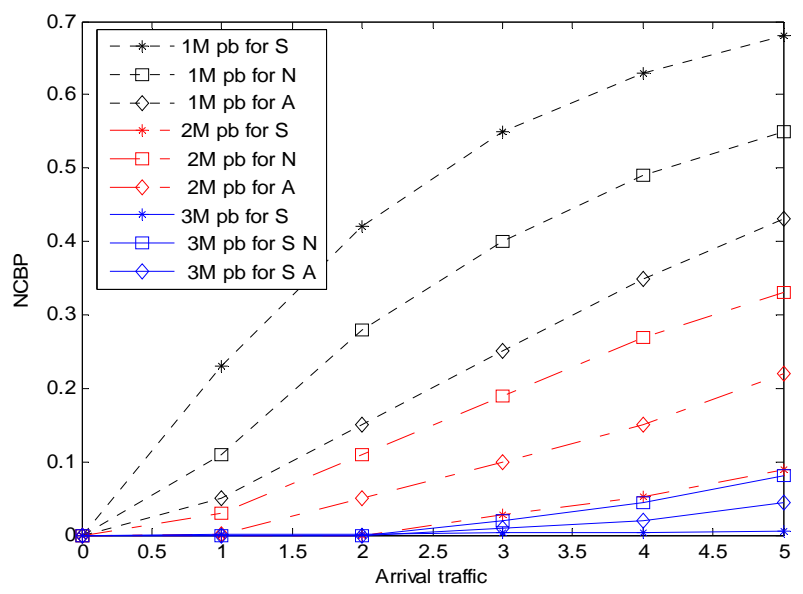

Figure 11. NCBP $(\mathrm{Pb})$ for class-1 calls with $1 \mathrm{M}: 2 \mathrm{M}: 3 \mathrm{M}=$ 50:25:25.

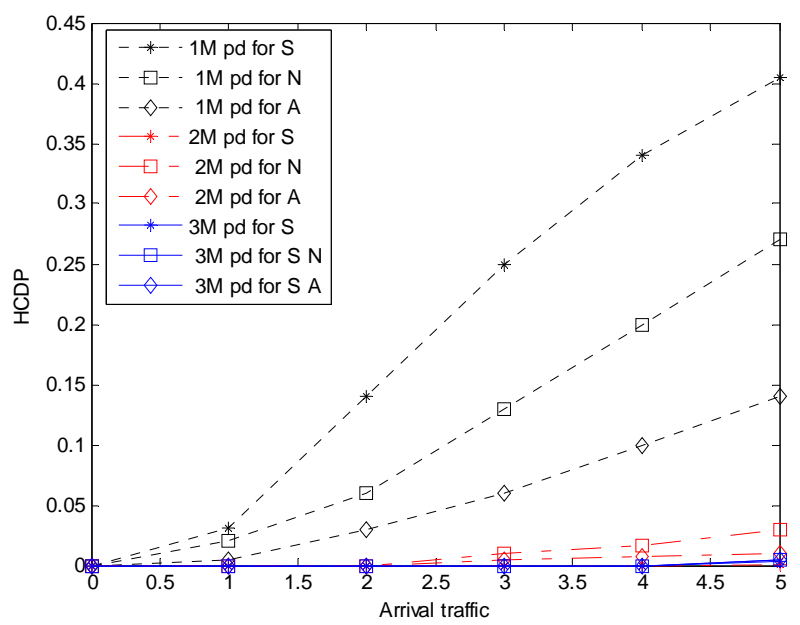

Figure 12. HCDP (Pd) for class-1 calls with $1 \mathrm{M}: 2 \mathrm{M}: 3 \mathrm{M}=$ 50:25:25.

the SJCAC schemes.

The HCDP for the ATJCAC scheme is always less than the corresponding HCDP for the NATJCAC scheme. Note that lower HCDP of the ATJCAC scheme implies that its connection-level QoS is better than that of the NATJCAC scheme. The reason why the HCDP of the ATJCAC scheme is less than the NATJCAC scheme is as follows. When the total $b b u$ allocated to handoff calls is being fully utilized, incoming handoff calls are rejected by the NATJCAC scheme whereas the ATJCAC scheme adapts (degrades) the bandwidth of some of the ongoing adaptive calls to free just enough $b b u$ to accommodate the incoming handoff calls. Consequently, the HCDP of the ATJCAC is less than that of the NATJCAC. However, an adaptive class- $i$ call is never degraded below the minimum $b b u$ necessary to guarantee its minimum QoS requirements. For SJCAC the Pd for one-mode terminals is very high whereas Pd for two-mode and three-mode terminals are very low. Thus, one-mode terminals are treated unfairly by the SJCAC scheme. 


\subsection{Second Scenario: $1 M: 2 M: 3 M=25: 50: 25$}

Figure 13 shows the effect of varying the call arrival rate on the NCBP $(\mathrm{Pb})$ of 1-mode, 2-mode, and 3-mode terminals for SJCAC, NATJCAC and the proposed ATJCAC. As shown in Figure 13, Pb for the three JCAC schemes and three classes of terminals increases with an increase in arrival rate for three JCAC schemes. This is expected. However, for 1-mode terminals, $\mathrm{Pb}$ of the NATJCAC scheme is lower than the corresponding $\mathrm{Pb}$ of the SJCAC schemes. The NATJCAC scheme is able to reduce the $\mathrm{Pb}$ by admitting most of the calls from 3-mode terminals into RAT 3, which has the lowest terminal support index, thereby reserving RAT 1 and RAT 2 for calls from 1-mode and 2-mode terminals, respectively. However, the reduction in 1 -mode terminals $\mathrm{Pb}$ for NATJCAC scheme is at the expense of the Pb1 of calls from 3-mode terminals. It can be seen that for 3-mode terminals, the $\mathrm{Pb}$ of the NATJCAC scheme is higher than the corresponding $\mathrm{Pb}$ of the SJCAC schemes.

The NCBP for the ATJCAC scheme is always less than the corresponding NCBP for the NATJCAC scheme. Note that lower NCBP of the ATJCAC scheme implies that its connection-level QoS is better than that of the NATJCAC scheme. The reason why the NCBP of the ATJCAC scheme is less than the NATJCAC scheme is as follows. When the total $b b u$ allocated to new calls is being fully utilized, incoming new calls are rejected by the NATJCAC scheme whereas the ATJCAC scheme adapts (degrades) the bandwidth of some of the ongoing adaptive calls to free just enough $b b u$ to accommodate the incoming new calls. Consequently, the NCBP of the ATJCAC is less than that of the NATJCAC. However, an adaptive class-i call is never degraded below the minimum $b b u$ necessary to guarantee its minimum QoS requirements.

For SJCAC the $\mathrm{Pb}$ for one-mode terminals is very high whereas $\mathrm{Pb}$ for two-mode and three-mode terminals are very low. Thus, one-mode terminals are treated unfairly by the SJCAC scheme.

Figure 14 shows the effect of varying the call arrival rate on the HCDP (Pd) of 1-mode, 2-mode, and 3-mode terminals for SJCAC, NATJCAC and the proposed ATJCAC. As shown in Figure 14, Pd for the three JCAC schemes and three classes of terminals increases with an increase in arrival rate for three JCAC schemes. This is expected.

However, for 1-mode terminals, Pd of the NATJCAC scheme is lower than the corresponding Pd of the SJCAC schemes. The NATJCAC scheme is able to reduce the Pd by admitting most of the calls from 3 -mode terminals into RAT 3, which has the lowest terminal support index, thereby reserving RAT 1 and RAT 2 for calls from 1-mode and 2-mode terminals, respectively. However, the reduction in 1-mode terminals Pd for NATJCAC scheme is at the expense of the $\mathrm{Pd} 1$ of calls from 3-mode termi-

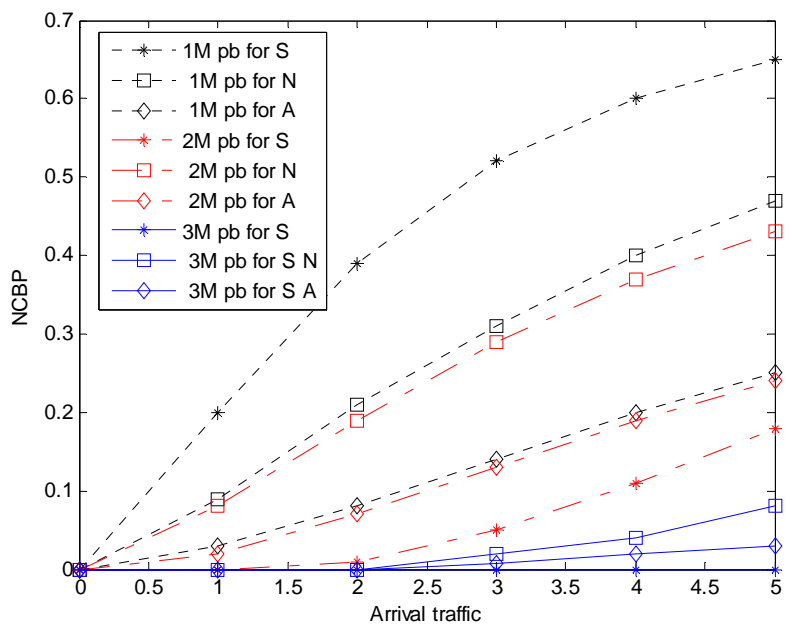

Figure 13. NCBP $(\mathrm{Pb})$ for class-1 calls with $1 \mathrm{M}: 2 \mathrm{M}: 3 \mathrm{M}=$ 25:50:25.

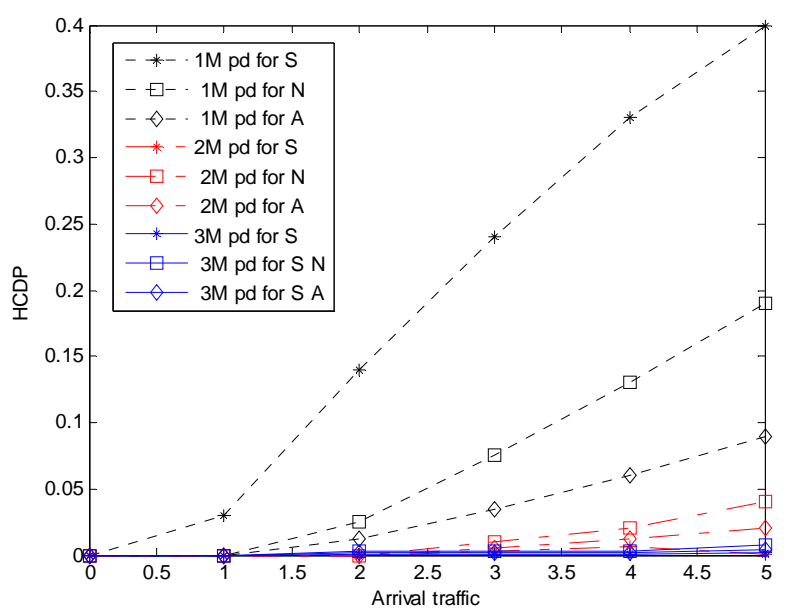

Figure 14. HCDP (Pd) for class-1 calls with 1M:2M:3M = 25:50:25.

nals. It can be seen that for 3-mode terminals, the Pd of the NATJCAC scheme is higher than the corresponding Pd of the SJCAC schemes.

The HCDP for the ATJCAC scheme is always less than the corresponding HCDP for the NATJCAC scheme. Note that lower HCDP of the ATJCAC scheme implies that its connection-level QoS is better than that of the NATJCAC scheme. The reason why the HCDP of the ATJCAC scheme is less than the NATJCAC scheme is as follows. When the total $b b u$ allocated to handoff calls is being fully utilized, incoming handoff calls are rejected by the NATJCAC scheme whereas the ATJCAC scheme adapts (degrades) the bandwidth of some of the ongoing adaptive calls to free just enough $b b u$ to accommodate the incoming handoff calls. Consequently, the HCDP of the ATJCAC is less than that of the NATJCAC. However, an adaptive class-i call is never degraded below the minimum $b b u$ necessary to guarantee its minimum QoS requirements. For SJCAC the Pd for one-mode terminals is 
very high whereas Pd for two-mode and three-mode terminals are very low. Thus, one-mode terminals are treated unfairly by the SJCAC scheme.

\section{Conclusion}

In heterogeneous wireless network, There is a need to make RAT selection decisions in addition to call admission decisions. In future wireless networks multimedia, traffic will have different QoS requirements. The adaptive-TJCAC scheme fair radio resource allocation, guarantees the QoS requirements of all accepted call and reduces both new call blocking probability and handoff call dropping probability in the heterogeneous wireless networks. It prioritizes handoff calls over new calls by making the handoff call rejection thresholds higher than the new call rejection thresholds. The proposed ATJCAC algorithm makes call admission decisions based on mobile terminal modality (capability), network load, adaptive the bandwidth of ongoing call and radio access technology (RAT) terminal support index. Performance of the adaptive-TJCAC scheme is compared with nonadaptive TJCAC scheme and SJCAC scheme. Simulation results show that the proposed ATJCAC scheme reduces call blocking/dropping probability.

\section{REFERENCES}

[1] O. E. Falowo and H. A. Chan, "Joint Call Admission Control Algorithms: Requirements, Approaches, and Design Considerations," Computer Communications, Vol. 31, No. 6, 2008, pp. 1200-1217. doi:10.1016/j.comcom.2007.10.044

[2] O. E. Falowo and H. A. Chan, "Joint Call Admission Control for Next Generation Wireless Network," IEEE Conference on Electrical and Computer Engineering, Ottawa, May 2006, pp. 1151-1154.

[3] S. Lee, K. Sriram, K. Kim, Y. Kim and N. Golmie, "Vertical Handoff Decision Algorithms for Providing Optimized Performance in Heterogeneous Wireless Networks," IEEE Transactions on Vehicular Technology, Vol. 58, No. 2, 2009, pp. 865-881. doi:10.1109/TVT.2008.925301

[4] S. S. Rappaport, "The Multiple-Call Hand-Off Problem in High-Capacity Cellular Communications Systems," IEEE Transactions on Vehicular Technology, Vol. 40, No. 3, 1991, pp. 546-557. doi:10.1109/25.97509

[5] M. Naghshineh and M. Schwartz, "Distributed Call Admission Control in Mobile/Wireless Networks," IEEE Journal on SAC, Vol. 14 No. 4, 1996, pp. 711-717.

[6] Y. Fei and V. Krishnamurthy, "Optimal Joint Session Admission Control in Integrated WLAN and CDMA Cellular Networks with Vertical Handoff," IEEE Transactions on Mobile Computing, Vol. 6, No. 1, 2007, pp. 126139. doi:10.1109/TMC.2007.250676

[7] X. G. Wang, G. Min and J. E. Mellor, "Adaptive QoS Control in Cellular and WLAN Interworking Networks,"
Second International Working Conference on the Performance Modeling and Evaluation of Heterogeneous Networks (HET-NETs'04), July 2004.

[8] D. Karabudak, C. Hung and B. Bing, "A Call Admission Control Scheme Using Genetic Algorithms," Symposium on Applied Computing (SAC 04), March 2004, pp. 11511158 .

[9] K. Murray and D. Pesch, "Policy Based Access Management and Handover Control in Heterogeneous Wireless Networks," The IEEE Vehicular Technology Conference, Vol. 5, 2004, pp. 3319-3323.

[10] K. Murray and D. Pesch, "Intelligent Network Access and Inter-System Handover Control in Heterogeneous Wireless Networks for Smart Space Environments," 1st International Symposium on Wireless Communication Systems, 2004, pp. 66-70.

[11] Q. Deng, D.-L. Xie, B. Hu, Y. Shi and S.-Z. Chen, "Joint Admission Control through Vertical Handoffs in Heterogeneous Wireless Network," Global Mobile Congress (GMC), Shanghai, 18-19 October 2010, pp. 1-5.

[12] X. Li, H. Ji, P. Si and L. Zhang, "Joint Session Admission Control Scheme in Integrated WLAN and 3G Networks," 5th International ICST Conference on Communications and Networking in China (CHINACOM 2010), August 2010, pp. 1-5. doi:10.4108/chinacom.2010.5

[13] M. Khabazian, O. Kubbar and H. Hassanein, "Call Admission Control with Resource Reservation for MultiService OFDM Networks," 2012 International Conference on Computing, Networking and Communications (ICNC), Maui, 30 January-2 February 2012, pp. 781-785. doi:10.1109/ICCNC.2012.6167530

[14] Z.-H. Liu and J.-C. Chen, "Design and Analysis of the Gateway Relocation and Admission Control Algorithm in Mobile WiMAX Networks," IEEE Transactions on Mobile Computing, Vol. 11, No. 1, 2012, pp. 5-18.

[15] X. Gelabert, J. Perez-Romero, O. Sallent and R. Agust1, "A Markovian Approach to Radio Access Technology Selection in Heterogeneous Multiaccess/Multiservice Wireless Networks," IEEE Transactions on Mobile Computing, Vol. 7, No. 10, 2008, pp. 1257-1270. doi:10.1109/TMC.2008.50

[16] O. E. Falowo, "Terminal-Modality-Based Joint Call Admission Control Algorithm for Fair Radio Resource Allocation in Heterogeneous Cellular Networks," International Journal of Communications, Network and System Sciences, Vol. 5, No. 7, 2012, pp. 392-404. doi:10.4236/ijens.2012.57049

[17] O. E. Falowo and H. A. Chan, "Joint Call Admission Control Algorithm for Fair Radio Resource Allocation in Heterogeneous Wireless Networks Supporting Heterogeneous Mobile Terminals," 7th Annual IEEE Consumer and Communication \& Networking Conference (IEEE CCNC), Las Vegas, 9-12 January 2010, pp. 1-5.

[18] W. Zhang, "Performance of Real-Time and Data Traffic in Heterogeneous Overlay Wireless Networks," Proceedings of the 19th International Teletraffic Congress, 29 August-2 September 2005.

[19] H. Holma and A. Toskala, "WCDMA for UMTS," 2nd Edition, John Wiley \& Sons, New York, 2001. 
[20] V. Pla, J. M. Gimenez-Guzmany, J. Martinez and V. Casares-Giner, "Optimal Admission Control Using Handover Prediction in Mobile Cellular Networks," Proceedings of the 2nd International Working Conference on Performance Modelling and Evaluation of Heterogeneous Networks (HET-NETs '04), 26-28 July 2004.

[21] G. Kesidis, J. Walrand and C.-S. Chang, "Effective Bandwidths for Multi-Class Markov Fluids and Other ATM Sources," IEEE/ACM Transactions on Networking, Vol. 1, No. 4, 1993, pp. 424-428. doi:10.1109/90.251894

[22] N. Nasser and H. Hassanein, "Dynamic Threshold-Based
Call Admission Framework for Prioritized Multimedia Traffic in Wireless Cellular Networks," Proceedings of the IEEE Global Telecommunications Conference (GLOBECOM'04), 29 November-3 December, 2004, pp. 644649.

[23] S.-P. Chung and J.-C. Lee, "Performance Analysis and Overflowed Traffic Characterization in Multi-Service Hierarchical Wireless Networks," IEEE Transactions on Wireless Communications, Vol. 4, No. 3, 2005, pp. 904918. doi:10.1109/TWC.2005.847031 